\title{
A New Call Admission Control Algorithm for VoIP in IEEE 802.11 Wireless LANs
}

\author{
Alex P. da Silva, Student Member, IEEE, Júlio F. Pimentel, Student Member, IEEE, Francisco R. P. \\ Cavalcanti, Member, IEEE, Vicente A. de Sousa Jr., Student Member, IEEE
}

\begin{abstract}
In the past few years, the IEEE 802.11 WLAN has become very popular and widely deployed for Internet access. On the other hand, voice over IP is one of the fast growing Internet applications today. Thanks to the convergence of these two trends, it is believed that VoIP over WLAN is expected to become an important Internet applications. In this context, the so called "avalanche effect" has been identified as a real problem in a WLAN network, when operating near its capacity limit, in which the admission of an additional call may result in unacceptable QoS for all the ongoing VoIP connections. Therefore, this work proposes a new call admission control mechanism, based on the access point's transmission buffer utilization ratio, to mitigate this problem. The obtained results show that the proposed mechanism mitigates the "avalanche effect", leading to stable service operation, acceptable QoS levels and significant capacity gains considering tested scenarios.
\end{abstract}

Index Terms-WLAN, VoIP, Call Admission Control.

\section{INTRODUCTION}

Supporting voice service over IEEE 802.11 Wireless LAN (WLAN) poses significant challenges, since the performance characteristics of the Medium Access Control (MAC) and Physical (PHY) layers are worse than that of the wired networks. In this way, the Voice over Internet Protocol (VoIP) applications over WLAN bring up some issues related to the system architecture, network capacity, admission control and QoS provision.

This contribution proposes an investigation of Call Admission Control (CAC) mechanism for WLAN systems supporting VoIP applications, aiming to indicate solutions to provide QoS for the new and ongoing VoIP connections. We have investigated the so called "avalanche effect" which indicates CAC as an interesting research issue. In this context, we have proceeded the performance evaluation of three CAC algorithms. Two of them were found in specialized literature and the other one represents the innovative proposal of this work.

This work is organized as follows. This section presents a brief description of the VoIP service and of the IEEE 802.11 WLAN. Section II discusses performance problems existing when VoIP is offered over WLAN along with the literature review. Existing and proposed CAC solutions are

This work is supported by a grant from Ericsson of Brazil Research Branch under ERBB/UFC.10 Technical Cooperation Contract. All authors are with Wireless Telecommunications Research Group (GTEL), at Teleinformatic Engineering Department (DETI), Federal University of Ceará, Fortaleza, Brazil, URL: http://www.gtel.ufc.br.(emails:\{alex, julio, rodrigo, vicente\}@gtel.ufc.br). Francisco Rodrigo P. Cavalcanti is partially supported by CNPq with a Researcher Scholarship, grant n. 304477/2002-8. Vicente A de Sousa Jr. is scholarship supported by FUNCAP. described in section III. In section IV, the system modeling and performance evaluation methodology are detailed. After that, the performance results are drawn. Finally, the conclusions are discussed in section VI.

\section{A. Voice over IP}

Traditionally, the main load of information transported through the public communication networks is voice. To provide this service, networks based on circuit switching are generally used. While these networks provide an adequate voice quality, they can be highly inefficient regarding the bandwidth utilization [1].

Still under the bandwidth context, networks using packet switching technology based on the Internet Protocol (IP) are more efficient. However, these networks need a more elaborated implementation to provide transport of voice. In this way, VoIP appears as a technology that allows to establish phone calls over an IP network (including the Internet), in order that the voice transmission becomes one more service supported by the data network. Then, the VoIP service aims to provide over the packet switching network, similar voice quality experienced over the circuit switching network.

Some of main advantages of VoIP service as compared to the traditional circuit-switched voice service include:

- Higher efficiency of the bandwidth utilization;

- Low cost softwares to PC (Personal Computer) and PDA (Personal Digital Assistant);

- Growing of broadband connections, including ones through WLAN hotspots;

- Creation of new services that combine voice communication with other media and data applications;

- Reduced cost of the call.

However, some disadvantages to the VoIP implementation include:

- High quality and reliability of the traditional voice network;

- Variability of the VoIP quality. This problem is more critical in wireless networks due to the higher packet loss, longer transmission delays and higher delay jitter;

The VoIP service uses the IP protocol to transmit voice as data packets over an IP network. In this way, a VoIP system can be implemented in any network based on IP protocol: Internet, intranets and local area networks. In these systems, the voice signal is digitalized, compressed and converted into IP packets before the effective transmission over the network. 


\section{B. IEEE 802.11 Network}

The IEEE 802.11 technology is at present the most popular alternative for wireless local area networks. Initially developed to provide low cost wireless connectivity without any QoS guarantees, the IEEE 802.11 standard families has been applied to both indoor and outdoor environments. Due to its convenience, mobility, low-cost and high-speed access, WLAN represents an important future trend for Internet access. In the following, we briefly review the main features of the IEEE 802.11a PHY and MAC layers [2].

1) IEEE 802.11a Physical Layer: The IEEE 802.11a standard uses Orthogonal Frequency Division Multiplexing (OFDM) modulation that, due to its robustness in frequency selective channels, it is an important transmission technique for future wireless communication networks.

The OFDM-based physical layer of the IEEE 802.11a standard implements 8 operation modes, as shown in table I, for transmission rates up to $54 \mathrm{Mbps}$. For each new transmission attempt, link adaptation is performed in order to select one of the 8 operation modes.

I: IEEE 802.11a rate-dependent parameters.

\begin{tabular}{l|l|l|l}
\hline \hline $\begin{array}{l}\text { Data rate } \\
(M b p s)\end{array}$ & Modulation & $\begin{array}{l}\text { Coding rate } \\
(R)\end{array}$ & $\begin{array}{l}\text { Bytes per OFDM } \\
\text { symbol }(B p S)\end{array}$ \\
\hline \hline 6 & BPSK & $1 / 2$ & 3 \\
\hline 9 & BPSK & $3 / 4$ & 4.5 \\
\hline 12 & QPSK & $1 / 2$ & 6 \\
\hline 18 & QPSK & $3 / 4$ & 9 \\
\hline 24 & 16 QAM & $1 / 2$ & 12 \\
\hline 36 & 16 QAM & $3 / 4$ & 18 \\
\hline 48 & 64 QAM & $2 / 3$ & 24 \\
\hline 54 & 64 QAM & $3 / 4$ & 27 \\
\hline
\end{tabular}

2) IEEE 802.11 WLAN MAC protocol: The IEEE $802.11 \mathrm{a} / \mathrm{b} / \mathrm{g}$ standards share the same MAC layer that uses Carrier Sense Multiple Access with Collision Avoidance (CSMA/CA) for contention data, a Request-to-Send/Clear-to-Send (RTS/CTS) mechanism to accommodate the hidden terminal problem, and an optional mechanism called Point Coordination Function (PCF) to support time-bounded applications.

The Distributed Coordination Function (DCF) mode is the mandatory mechanism and is based on CSMA/CA and binary exponential backoff. The PCF mode is optional access mechanism, which is polling-based, with a central element, the Point Coordinator (PC), controlling and giving permission of to the stations (STAs) to transmit.

In the basic access mechanism of the DCF, a station with a frame to transmit monitors the medium during an idle interval equal to a Distributed Interframe Space (DIFS). If the medium is sensed idle by the station, it transmits a frame immediately. Otherwise, the station waits for a random backoff interval before transmitting, and afterwards it transmits a frame. If another transmission is detected on the channel, the transmission is deferred and the backoff timer of the station is frozen. The backoff timer is reactivated when the channel is sensed idle again for more then a DIFS time. Figure 1 shows the operation of the basic access mechanism.

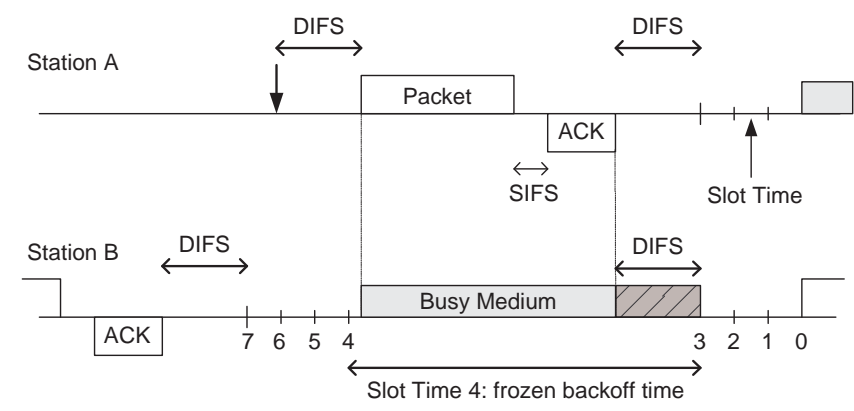

1: Basic access mechanism.

Having received a frame correctly, the destination station waits for a SIFS interval immediately following the reception of the data and transmits an ACK back to the source station, indicating that the frame has been received correctly. If the source station does not receive an ACK, the frame is assumed to be lost and the source station schedules retransmission with a new random backoff interval that can be larger than the previous one.

\section{LiterATURE REVIEW: VOIP PERFORMANCE PROBLEMS OVER WLAN NETWORKS}

The IEEE 802.11a supports transmission rates up to 54 Mbps. On the other hand, a VoIP session requires nearly 64 kbps when using the G.711 Codec [3], a naive calculation might indicate that the IEEE 802.11a network can support up to $54 M / 64 k=844$ flows, which corresponds to 422 full-duplex VoIP sessions.

However, [3]-[7] show that the maximum capacity of VoIP connections held on an IEEE 802.11a network is limited to some tens of sessions, due to overheads introduced by protocol headers (IP/UDP/RTP/MAC/PHY headers), backoff times, transmission of acknowledge packets (ACK) and intervals of packet transmissions.

Besides the low capacity, studies performed in [3], [8] revealed that placing an additional call that exceeds the capacity of the wireless network will result in unacceptable call quality for all ongoing VoIP sessions. In this work, we call this degradation phenomenon "avalanche effect". The "avalanche effect" occurs due to the unbalanced traffic between the downlink and uplink flows. In this situation, the access point (AP) is no longer able to delivery the packets in time and gets curtailed, which leads to unaccepted packet loss for all VoIP streams transmitted from the access point to STAs resulting in bad call quality for all connections.

The limited capacity of the WLANs to provide real-time services like VoIP is a problem that was extensively studied in several works. In [9], [10], the PCF mechanism was used to provide real-time services in WLANs. However, the PCF is not supported by most of the equipments available in the market. In [11]-[13], the DCF mechanism was used in order to investigate several strategies to improve the VoIP capacity by changing the MAC protocol supported into the STAs. Changes in the MAC, however, could lead to incompatibility problems.

Instead of proposing manners to increase the WLAN capacity for the VoIP service, this work aims to evaluate 
mechanisms to assure, in an efficient way, the functioning of the system within a determined threshold to avoid the "avalanche effect". In this context, the implementation of an admission control mechanism becomes necessary since it can control the entrance of users in order to avoid the overall degradation of the network.

Some works describe admission control schemes for WLAN networks. However, the most of them is dedicated to the IEEE 802.11e standard, which has not been made commercial at the time of this writing. Some admission control proposals for this standard can be found in [14]-[22].

Only four admission control schemes are dedicated to the IEEE $802.11 \mathrm{a} / \mathrm{b} / \mathrm{g}$ MAC WLAN standard [8], [23]-[25]. In [23], the scheme is based on an equation that determines the maximum number of VoIP clients that an AP can support. In [24], a channel utilization rate is adopted as admission control metric. These two admission control mechanisms will be evaluated in this work and compared to the proposed algorithms. Modifications at the MAC layer are performed in [8], [25] for admission control, therefore these works were not considered to evaluation.

In face of the reduced number of works related to the admission control for VoIP service over IEEE 802.11 WLAN networks, we have identified this subject as a niche to be explored.

\section{Existing ANd Proposed CALl Admission CONTROL ALGORITHMS}

This section presents the three CAC algorithms previously mentioned. Two of them were found in the specialized literature [23], [24]. The third one corresponds to the main contribution of this work.

\section{A. CAC Algorithm Based on a Theoretical Network Capacity Estimation (EQA)}

This algorithm is based on a theoretical equation, provided by [23], to compute the maximum number of VoIP connections a single AP can support. This number depends on the maximum throughput the channel can achieve, which is a function of the packet size. Others factors affecting the channel throughput include the byte overheads of the RTP, UDP and IP protocols, MAC and PHY layers and all those due to the channel access mechanism, such as backoff procedures and interframe intervals (DIFS and SIFS).

Initially, we may express the channel throughput as

$$
\frac{T_{P}}{T_{P}+T_{\text {overhead }}} \cdot R_{\text {avg }}
$$

where $T_{P}$ is the time taken to transmit the VoIP payload of $P$ bytes and $T_{\text {overhead }}$ denotes the average overhead per RTP packet. For G.711 CODEC, this payload is 160 bytes for $20 \mathrm{~ms}$ of audio. $R_{a v g}$ is the average data transmission rate of the access point, which varies from $6 \mathrm{Mbps}$ to $54 \mathrm{Mbps}$ depending on the spacial distribution of the STAs.

$T_{\text {overhead }}$ can be divided into two components, the overhead incurred in transmitting the extra bytes of various networking layers, $T_{\text {layers }}$, and the overhead incurred by the MAC protocol. The later comprises one SIFS, one DIFS, the transmission time of one ACK $\left(T_{S I F S}, T_{D I F S}\right.$ and $T_{A C K}$, respectively) and the average idle time slots $\left(T_{d c f}\right)$ per frame as seen on the channel. The transmission time of a single VoIP packet taking into account all the overheads is shown in figure 2. According to [23], $T_{d c f}$, in microseconds, is given by

$$
T_{d c f}=4,5 \times 9+0,06 \times\left(T_{\text {total }}\right)
$$

where

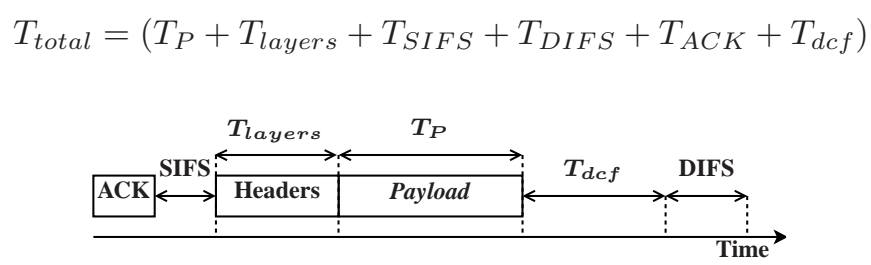

2: Transmission time of a single VoIP packet taking into account all the overheads.

Table II shows the overhead per packet, in bytes or microseconds, taking into account the average data rate.

II: Overhead per sent frame taking the average data rate into

\begin{tabular}{c|c|c}
\multicolumn{3}{|c}{ account. } \\
\hline \hline Overhead & Bytes & Time $(\mu \mathrm{s})$ \\
\hline \hline RTP Layer & 12 & $12 * 8 / R_{\text {avg }}$ \\
\hline UDP Layer & 8 & $8 * 8 / R_{\text {avg }}$ \\
\hline IP & 20 & $20 * 8 / R_{\text {avg }}$ \\
\hline MAC Header & 34 & $34 * 8 / R_{\text {avg }}$ \\
\hline PHY Header & & $32 \mu \mathrm{s}$ \\
\hline SIFS & & $16 \mu \mathrm{s}$ \\
\hline DIFS & & $34 \mu \mathrm{s}$ \\
\hline ACK & 14 & $18.7 \mu \mathrm{s}$ \\
\hline \hline
\end{tabular}

Due to the symmetric behavior of the VoIP connections, the AP receives and send packets to each of the $n$ STAs. Assuming G.711 CODEC, the AP handles uplink traffic of $n \times 64 \mathrm{kbps}$ and sends $n \times 64 \mathrm{kbps}$ downlink traffic. In this way, the total channel throughput is $n \times 128 \mathrm{kbps}$. Therefore, the maximum number of VoIP connections is given by

$$
n_{\max }=\frac{T_{P} \times R_{a v g}}{128000 \times\left(T_{\text {total }}+T_{d c f}\right)}
$$

From $n_{\max }$, the admission mechanism is very simple. Once reached the maximum number of VoIP connections, determined by equation (3), no more connections are admitted.

\section{B. Algorithm Based on the Channel Busyness Ratio (CBA)}

The channel busyness ratio $R_{b}$ is the fraction of time the medium is busy to the total time. According to [24], when the WLAN works at the "optimal point", the collision probability is small and $R_{b}$ is relatively stable around 0.90 . Let $B_{U}$ denote the channel utilization corresponding to this point. In [24], the authors have also introduced a bandwidth reservation for voice traffic as a percentage of $B_{U}$. However, in this work, we 
consider a scenario composed by VoIP traffic only. Therefore, the CAC algorithm should keep $R_{b}$ below $B_{U}$ to guarantee a good QoS level for all the ongoing calls.

The CAC mechanism admits a new voice call only if the requested resource is available. The bandwidth requirement of a voice call is characterized by three parameters, $\left(r, r_{\text {peak }}, l\right)$, where $r$ is the average transmitting rate, $r_{\text {peak }}$ is the peak rate, both in bits per second, and $l$ is the average packet length in bits. However, to conduct the CAC, the traffic rate needs to be converted to the channel utilization $u$ [24],

$$
\begin{aligned}
u & =\frac{r}{l} \times T_{\text {suc }}, \\
u_{\text {peak }} & =\frac{r_{\text {peak }}}{l} \times T_{\text {suc }},
\end{aligned}
$$

where $T_{\text {suc }}$ is the transmission time of one packet, including all the overheads.

The total bandwidth occupied by all admitted voice flows is recorded by the AP in two parameters $\left(u_{A}, u_{\text {peak } A}\right)$. They are updated when a voice flow joins or leaves through the following CAC procedure.

When trying to connect to the WLAN, the STA must send its channel utilization requirement to the AP. The AP examines whether there is enough resource to accommodate the new flow by means of the following procedure:

- If $u_{A}+u \leq B_{U}$ and $u_{\text {peakA }}+u_{\text {peak }} \leq B_{U}$, the AP accepts the current request and updates $\left(u_{A}, u_{\text {peakA }}\right)$ with $\left(u_{A}+u, u_{\text {peakA }}+u_{\text {peak }}\right)$.

- Otherwise, the AP rejects the request.

\section{New CAC algorithm based on the transmission buffer utilization ratio (BSA)}

The CAC algorithm based on the AP's transmission buffer utilization ratio is a simple and innovative proposal of this work. For each new connection request, the AP examines the buffer utilization ratio to control the admission of the new users. In this way, the algorithm monitors specifically the network's bottleneck in order to mitigate the "avalanche effect".

The formal admission rule for the proposed algorithm is stated as follows:

$$
\text { Admission }= \begin{cases}\text { Accepted, } & \text { if } U_{\text {buffer }} \leq L_{B U} \\ \text { Denied, } & \text { if } U_{b u f f e r}>L_{B U}\end{cases}
$$

where $U_{\text {buffer }}$ is the AP's transmission buffer utilization ratio defined as the ratio of the current buffer occupancy (in MAC-layer packets) to the total buffer space and and $L_{B U}$ is the blocking threshold for this ratio.

The motivation for developing this algorithm comes from the fact that the time behavior of the AP's transmission buffer utilization is quite similar to the average FER, the more representative QoS metric for the VoIP service, as shown in figure 3 . We may observe the abrupt rise on the measured average FER corresponding to the peaks in the number of connected users to the system. This sudden degradation characterizes the so called "avalanche effect".
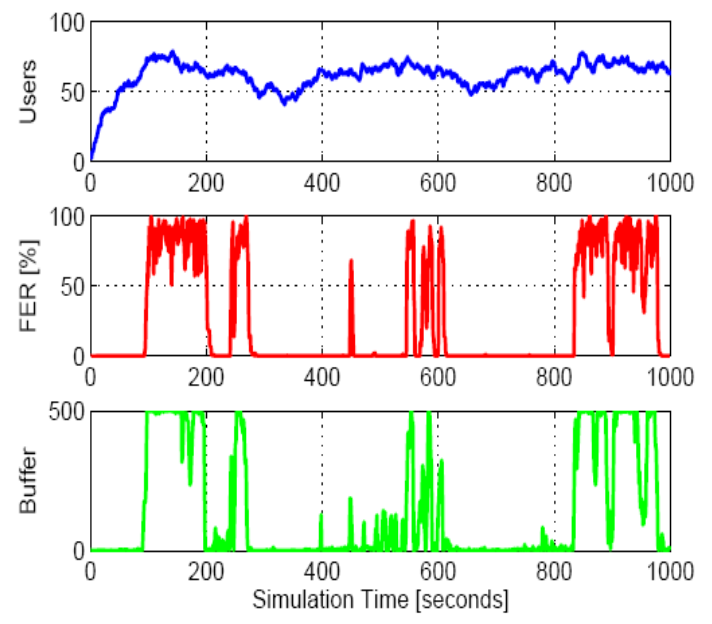

3: Time behavior of the system with no CAC mechanism.

A zoom of a FER peak, presented in figure 4, shows an even more interesting characteristic of the AP's buffer utilization. The effect of the "avalanche effect" on the buffer utilization is felt before the degradation of the average FER (behavior observed in all tested simulations). This way, the BSA algorithm may act blocking new connection before any degradation occurs in the network.
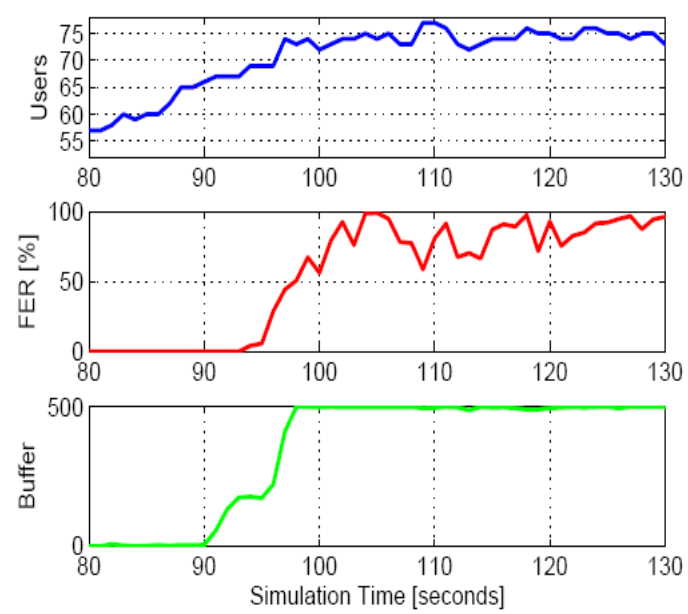

4: Detail of the time behavior of the system with no CAC mechanism.

\section{SyStem MODELING}

\section{A. Simulation Tool}

The WLAN Simulator developed and used in this work, is a dynamic event-driven system-level simulator based on the IEEE 802.11 standard [2]. This standard defines a common MAC layer for all IEEE 802.11 commercial products (e.g. 802.11a, 802.11b and 802.11g) and no QoS guarantees.

The functional structure of the WLAN simulator is presented in figure 5. Data input corresponds to the 
configuration parameters which are set in order to configure and control the simulations execution, e.g., placement of the access points, users birth rate, mobility and traffic profiles, MAC and PHY parameters, and so on. The block WLAN System controls the event manager, the access points and its stations. The event manager is the main functional entity of the WLAN simulator. It is responsible for handling the flow of the various events at the right time during the whole simulation.

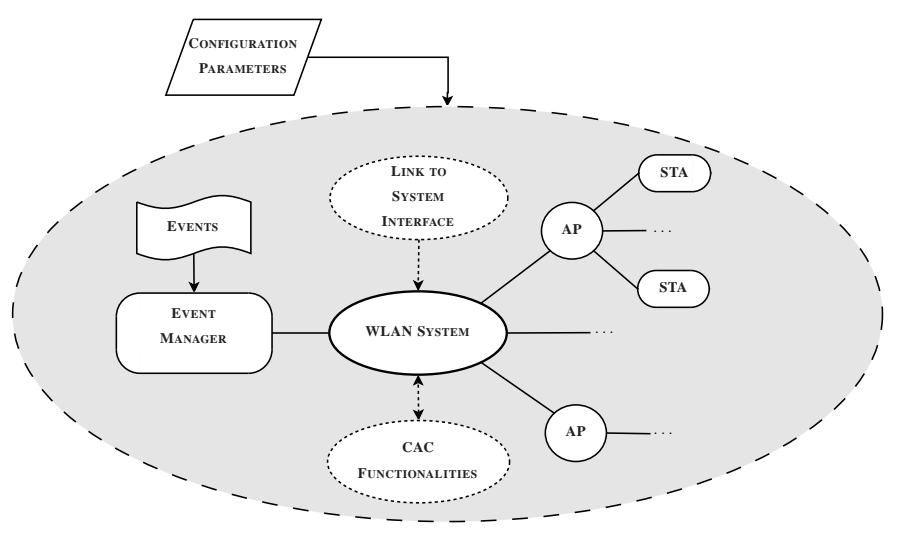

5: WLAN Simulator Functional Structure.

The event manager is illustrated in figure 6. The events are organized into a list and they are ordered in terms of their time stamps. The event manager fetches the first event, i.e., that one with the smallest time stamp, and transfers control to the corresponding event routine to update the system state appropriately. After that event's self-execution routine, a new event is likely to be generated. The event manager is then in charge of inserting this newly generated event in the ordered list of events. Since each event is self-executable, the event manager is not concerned about the kind of event that is being processed. This characteristic provides great flexibility to the event manager because no changes are required in its functioning in case a new kind of event is to be introduced in future versions of the simulator.

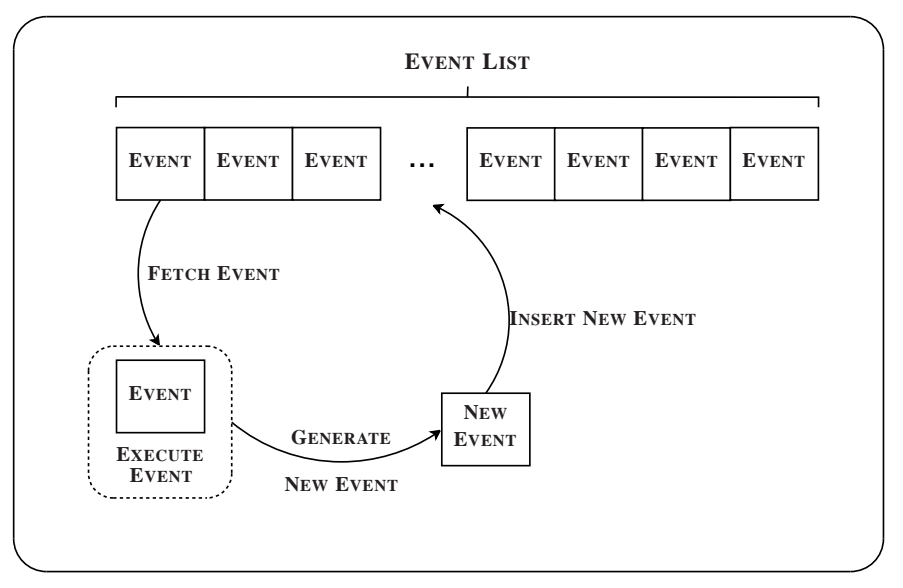

6: Event Manager.

\section{B. VoIP Modeling}

The VoIP traffic model is based on the two-state voice activity model as described in [26]. The VoIP call is composed by activity and silence periods with equal probability. The call arrives according to a Poisson process and its duration follows an exponential distributed random variable.

The G.711 codec has been chosen due to its simplicity and popularity. It supports coded voice at $64 \mathrm{kbps}$ during activity periods. The time between two continuous frames is $20 \mathrm{~ms}$, which corresponds to a rate of 50 frames/s. The payload size for this codec is $64000 /(50 \times 8)=160$ bytes.

In this work, we consider only the downlink direction for performance evaluation. It is assumed that during the activity period of an uplink user, its corresponding downlink user is in the silence period, i.e., the two traffics in the communication link are perfectly matched.

The RTP [27], UDP [28] and IP [29] protocols were not explicitly implemented in the WLAN simulation tool, but their influence was considered by the addition of the corresponding headers to the VoIP frame. No header compression scheme was used in this work.

According to [30], [31], the one-way mouth-to-ear delay of a VoIP frame should be less than $150 \mathrm{~ms}$. In our simulations, we consider a maximum delay of $100 \mathrm{~ms}$, which corresponds to the access delay and a fixed delay of $50 \mathrm{~ms}$ attributed to the other delays (e.g. from the fixed network). The access delay is defined as the time between the arrival of a VoIP frame at the AP and its successful transmission. If a frame arrives in its destination after $100 \mathrm{~ms}$, it is discarded.

The maximum Frame Error Rate (FER) should be around $1 \%$ to $3 \%$ to assure the quality of voice at the receiver [24]. In this work, we have considered a value of $2 \%$. This parameter defines the user satisfaction, which is described in the section IV-J.

\section{WLAN Scenario Layout}

We evaluate the CAC algorithms over a single AP scenario and all stations are uniformly distributed in the hotspot coverage area.

\section{MAC Modeling}

The simulator accounts for the basic access mechanism of the DCF, which is the fundamental mechanism to access the medium defined by the standard. This random access scheme employs CSMA/CA.

We have also consider that all ACK packets are correctly received. This supposition is quite reasonable since we consider that the ACK packets are transmitted by using the most error-robust OFDM mode, i.e., that one with the smallest transmission rate (6 Mbps).

\section{E. Interference}

The IEEE 802.11a WLAN [32] operates in the $5 \mathrm{GHz}$ band, which is free of interference from others equipments [33], [34]. Moreover, this standard allows that a bigger number of APs can coexist in the same area without interfering to each other 
because it provides non-overlapping channels, where each channel is associated to an AP. In [35], studies showed that the capacity of the IEEE 802.11a WLAN network composed by multiple APs is 8 times bigger than the one-AP IEEE 802.11 b capacity, due to the reduced co-channel interference. In addition, our network is composed of one AP. Based on these arguments, we do not consider any external interference source nor co-channel interference caused by adjacent access points.

\section{F. Propagation Model}

In our simulation, the free-space propagation model is used for the first 10 meters from the transmitter. After this distance, the propagation is modeled by the following path-loss formula [36]

$$
L(d)=L_{\text {free }}\left(d_{0}\right)+10 \cdot n \cdot \log \left(d / d_{0}\right)[d B]
$$

where $n=3.0, d_{0}=10 \mathrm{~m}$, and $L_{\text {free }}$ is free space propagation loss.

The shadowing is superimposed to the path loss effect and reflects local variations on the received power due to large scale obstacles. In the WLAN simulation, the shadowing component is normally distributed with $\sigma=4 d B$ [37] and decorrelation distance of $10 \mathrm{~m}$. The nominal AP radius is $60 \mathrm{~m}$, where a shadowing margin was added corresponding to $99 \%$ of the coverage area.

\section{G. Link Adaptation}

The OFDM mode is selected based on the perceived SNR, and consequently, determines the transmition bitrate. It means that, for a given SNR, we can find out the OFDM mode that will achieve the highest throughput, and thus, establish the bitrate for the current transmission based on a look-up table generated from the data shown in figure 7 . It can be noted that the throughput reaches its maximum value at about $30 \mathrm{~dB}$ of SNR.

The link adaption curves shown in figure 7 were obtained from a Physical-layer simulation tool, developed separately. These curves supplies link-level inputs for the WLAN system-level simulator.

\section{H. Wireless Channel Considerations}

In our simulator, we assumed the same propagation delay time for all stations in the system and it is taken equal to the delay observed by a station placed at the edge of the cell. This pessimistic approach leads to a delay $\delta=0.2 \mu \mathrm{s}$ for a $60 m$ hotspot radius. We also do not consider the existence of hidden terminals nor capture effects.

The Packet Error Rate (PER) for each VoIP frame transmission attempt is based on perceived SNR of each station. Figure 8 maps the PER for all OFDM operation modes. These curves were also obtained from the Physical-layer simulator, mentioned in section IV-G. The transmission of the acknowledgment frame is assumed as error free.

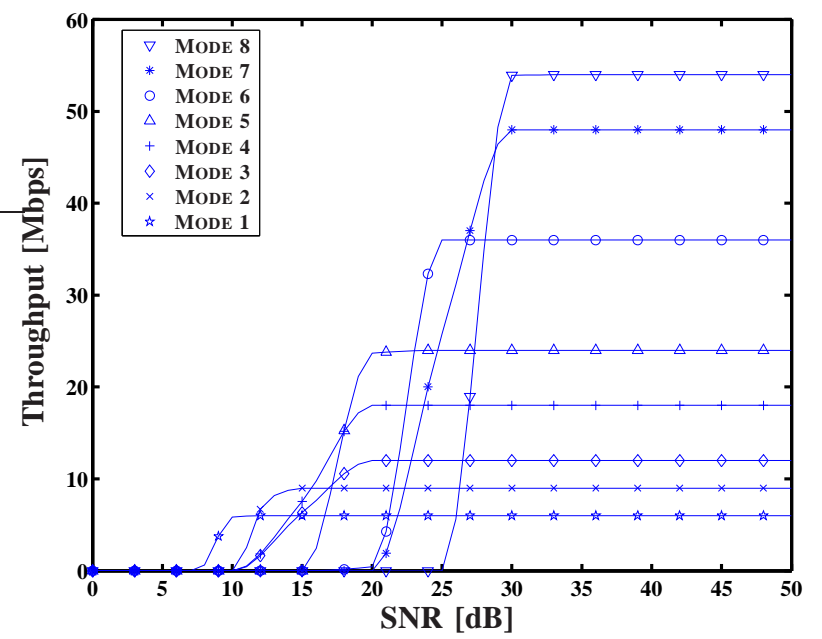

7: Link adaptation curves to OFDM PHY layer.

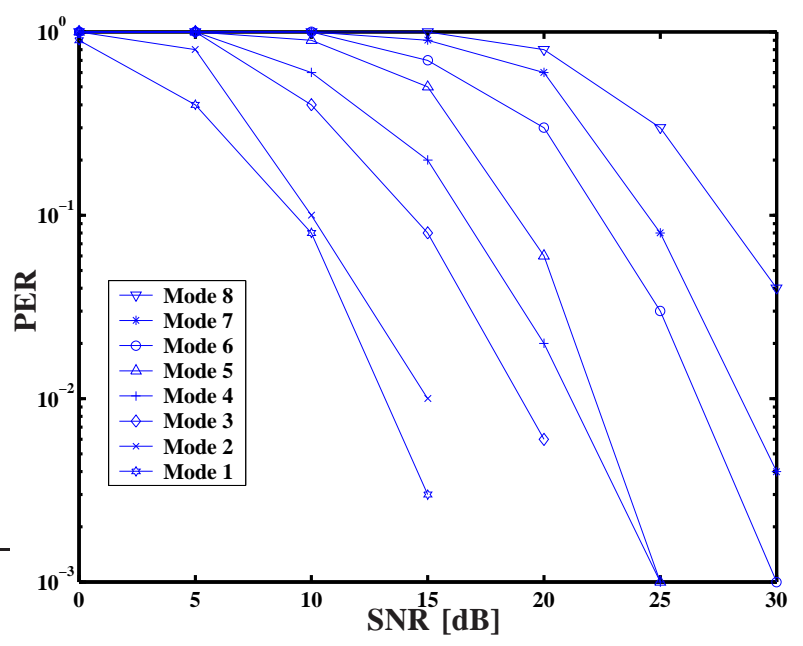

8: Packet Error Rate.

\section{Mobility}

In our simulator, the user speed and trajectory direction are not changed, i.e. the user remains in the same position during the whole simulation.

\section{J. Performance Metrics}

The main performance metrics used in this work are presented as follows.

- Percentage of Satisfied Users: A user is considered satisfied when it is connected to an AP and its access delay and FER are less than $100 \mathrm{~ms}$ and $2 \%$ respectively, as described in section IV-B. Otherwise, the user is considered unsatisfied. The percentage of satisfied users is defined as the ratio of the satisfied users to the total of users offered to the system during all simulation time.

- User Blocking Rate: It corresponds the ratio between the number of blocked users and the number of birth ones during the simulation. A blocked user is considered unsatisfied.

- System Capacity: The system capacity is based on a 
limit. In this work, this limit was defined as the load in which the system operates with $90 \%$ of satisfied users.

\section{- Offered Load}

The offered load is generally defined in terms of the arrival rate of new users in the system. However, in order to become a more representative and intuitive metric, the arrival rate of users was multiplied by the average voice call time. Therefore, the offered load is given by

$$
\frac{\text { Total of Birth Users }}{\text { Total Time }} \cdot \text { Average Session Time }
$$

The offered load to the AP is expressed in terms of the number of users per AP. Thus, this metric indicates the average number of simultaneous users offered to the AP.

\section{RESULTS}

Now, we present the system performance results for the evaluated CAC algorithms. Basically, we show performance of existing EQA and CBA algorithms and compare their performance with our CAC solution.

\section{A. Performance Evaluation of EQA and CBA Algorithms}

The downlink average access delay and the downlink average FER for both algorithms are shown in figures 9 and 10 , respectively.

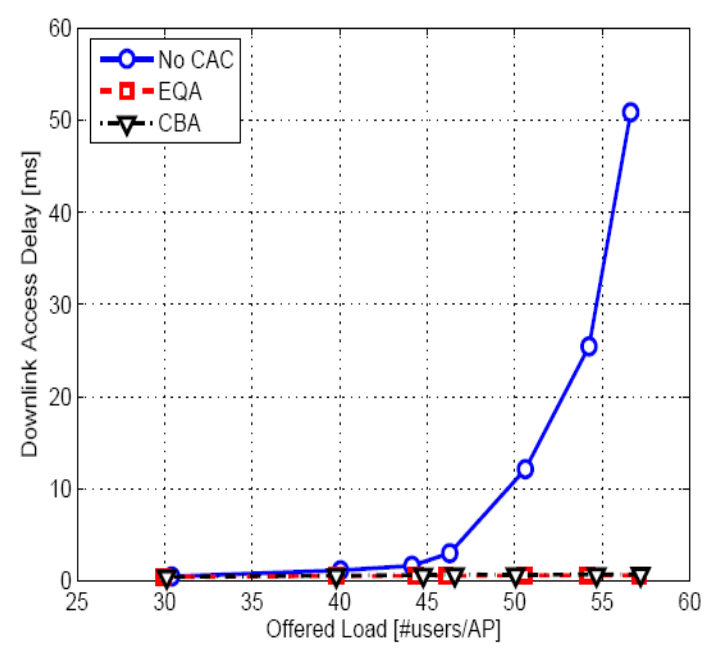

9: Downlink average access delay.

It can be noted that both algorithms present small values of FER and access delay for the connected users. The use of these algorithms have almost eliminated the so called "avalanche effect". This way, both EQA and CBA easily fulfill the main goal of a CAC algorithm, which is to protect the ongoing calls.

On the other hand, however, the user blocking rate is very high, as illustrated in figure 11. This leads to a low utilization of the network and waste of resources.

Finally, the user satisfaction curves are shown in figure 12. We observe that the capacity limit for both the EQA and the CBA are well below the reference curve, in which no CAC

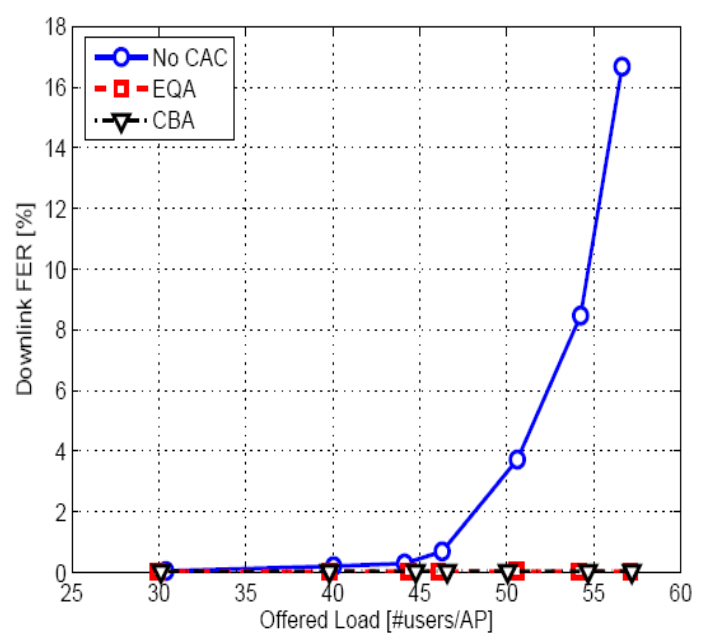

10: Downlink average FER.

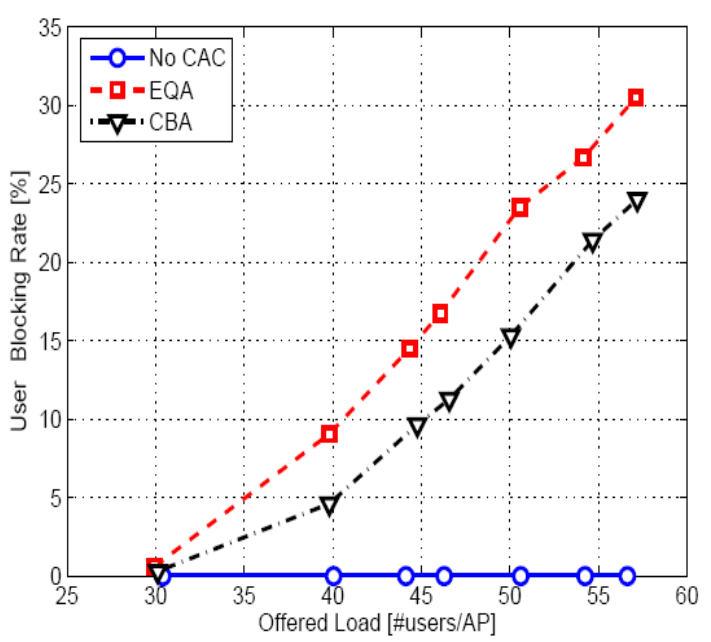

11: User blocking rate.

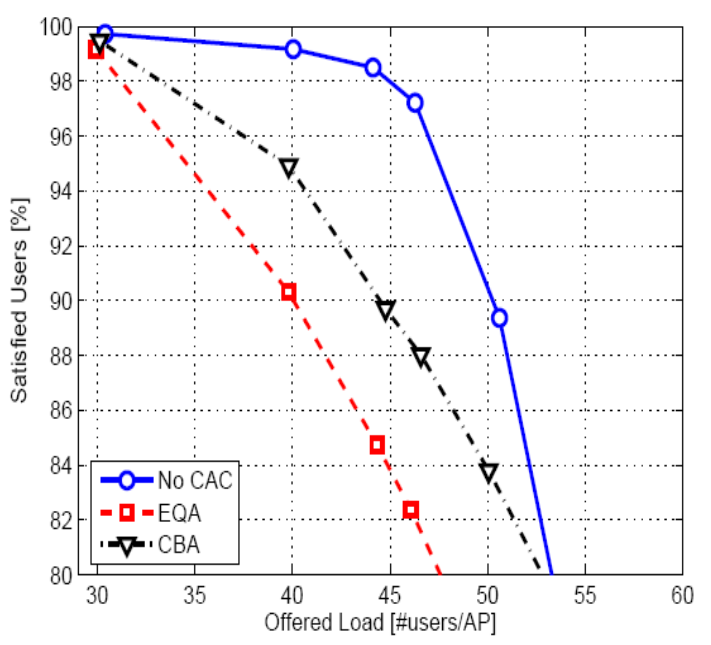

12: Percentage of satisfied users. 
mechanism was used. This behavior results from the very high blocking rate of both algorithms.

In summary, these algorithms provide very poor performance in terms of system capacity. Both the algorithm based on the theoretical network capacity estimation and the algorithm based on the channel busyness ratio are highly conservative in the light of the resource utilization. These algorithms allocate too much resources to the flows, overprotecting the ongoing calls and causing the network's sub-utilization, without taking profit of the VoIP's inherent intermittence.

\section{B. Performance Evaluation Comparison of the Proposed Algorithm}

An illustration of the WLAN network's time behavior is show in figures 13,14 e 15 .
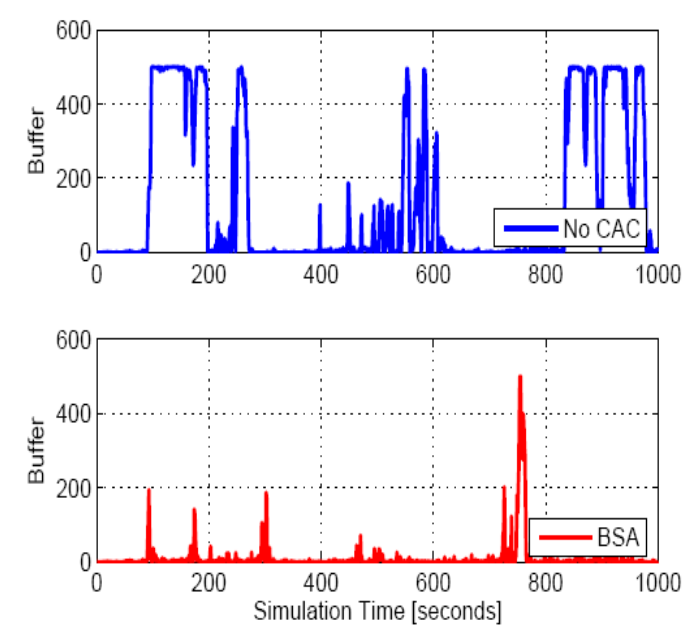

13: Transmission buffer utilization time behavior.
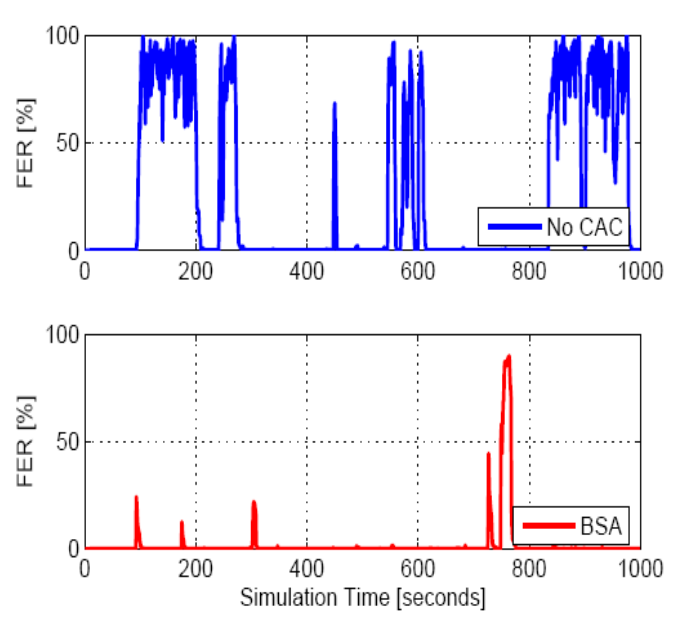

14: Average downlink FER time behavior.

These figures corresponds to the metrics of the AP's transmission buffer utilization, the average downlink FER and
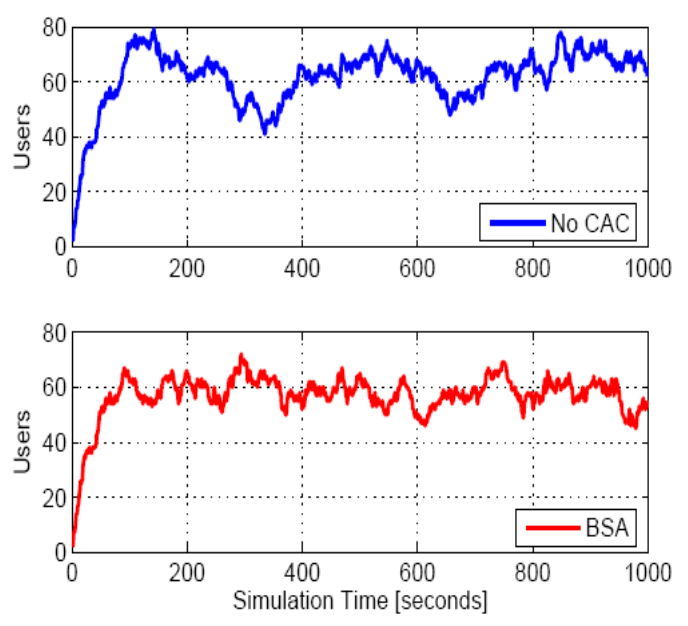

15: Number of connected users time behavior.

the number of connected users, respectively. Each figure is composed by two curves. The top curve shows the metric's time behavior when no CAC algorithm is used and the bottom curve corresponds to the given metric when the network make use of the BSA. In order to ensure the same statistical characteristics, we have used the same random seed for the two cases. The blocking threshold and the buffer size are $2 \%$ and 500 packets respectively. We have offered a relatively high load to the system of about 65 users/AP to aim for emphasize the action of the CAC mechanisms evaluated.

In figure 14 we may clearly observe that the use of the BSA leads to a significant reduction in the peaks of the measured average downlink FER, strongly reducing the "avalanche effect". We also observe that the remaining peaks on average downlink FER have reduced duration.

The effect of the use of the BSA in the number of connected users in emphasized in figure 15. This algorithm smooth the offered load by eliminating the peaks in the number of connected users to the system.

The performance evaluation for BSA has been carried out for five different values of the blocking threshold $L_{B U}: 1 \%$, $2 \%, 5 \%, 10 \%$ and $20 \%$.

Moreover, it is also important to verify the algorithm's dependency in the transmission buffer storage capacity. A buffer too small may discard, due to the lack of storage capacity, useful packets that possibly would arrive in time at the receiver. On the other hand, besides the device's physical limitation, a buffer too large may load the queue with too old packets that would certainly be discarded by the playout buffer at the receiver.

In order to capture the influence of the transmission buffer storage capacity in the performance of the BSA, the performance evaluation of this algorithm has been performed for three different buffer sizes: 300, 500 and 1000 packets.

Figures 16, 17 and 18 show the measured downlink average FER for the three values of the AP's transmission buffer.

The increase of the average FER with the offered load is a common overall behavior in the curves presented. In the 


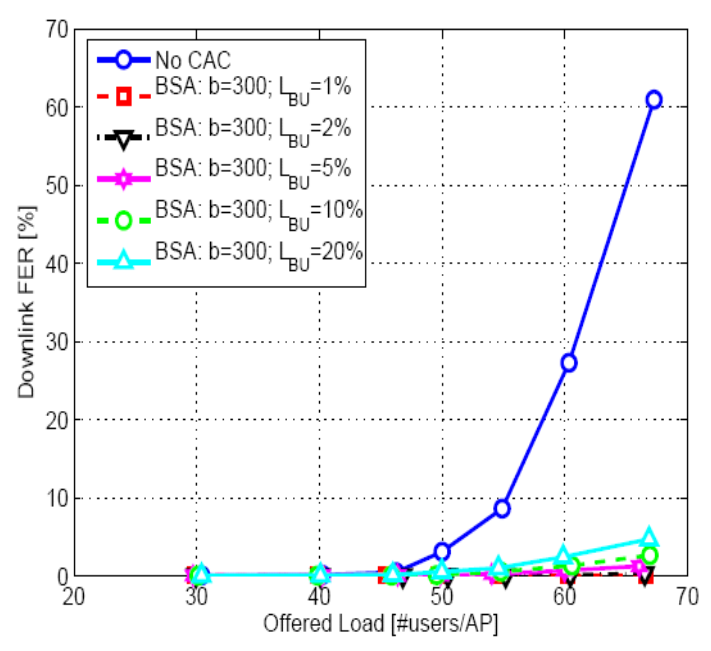

16: Downlink average FER, buffer capacity of 300 packets.

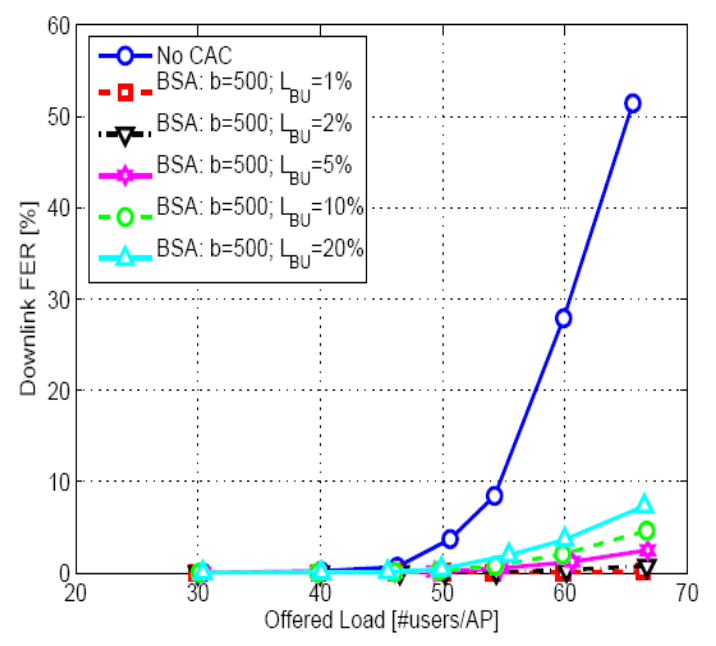

17: Downlink average FER, buffer capacity of 500 packets.

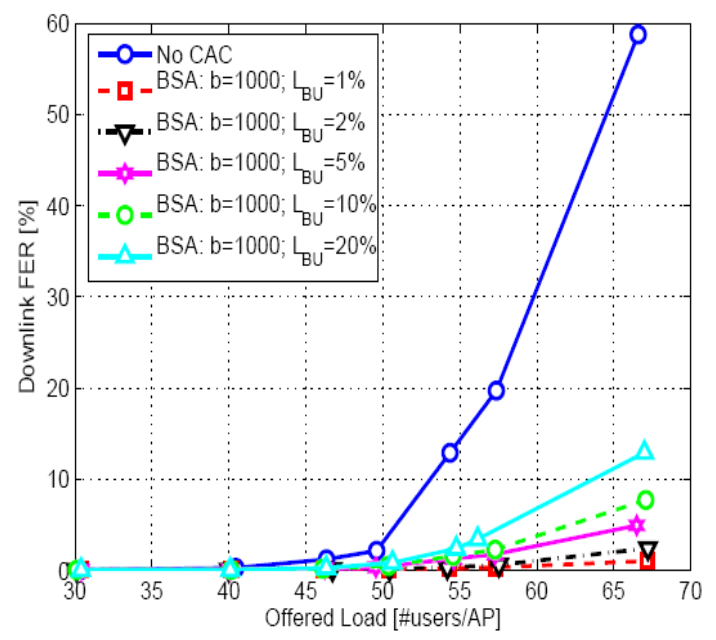

18: Downlink average FER, buffer capacity of 1000 packets.

light of the blocking threshold, we observe basically the same behavior in the three figures. The curves for the less restrictive blocking thresholds corresponds to higher values of average FER. This happens because the higher the blocking threshold the later the blocking mechanism of the CAC will be triggered and more overloaded will be the whole network.

When comparing the three figures, we see that the average FER increases with the AP's transmission buffer storage capacity. That is, the smaller values of average FER were observed for a 300-packet buffer, while the 1000-packet buffer shows the worst performance in terms of FER.

The user blocking rates are presented in the figures 19, 20 and 21 .

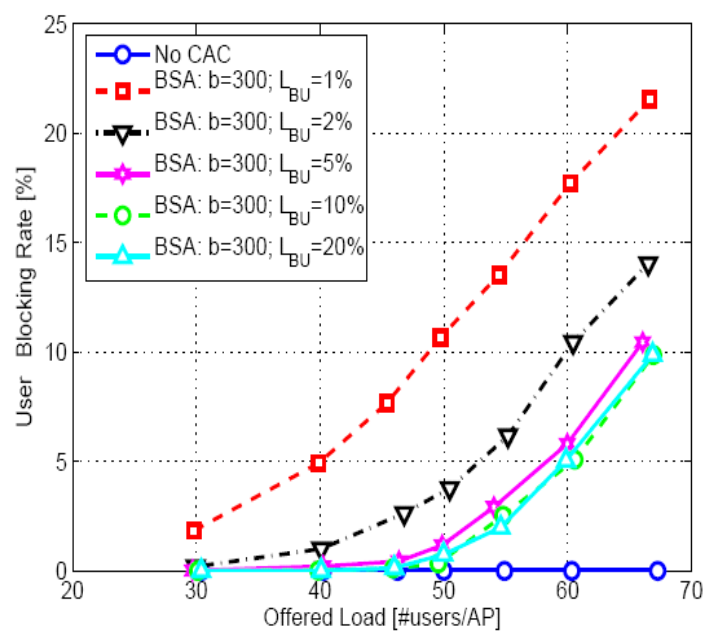

19: User blocking rate, buffer capacity of 300 packets.

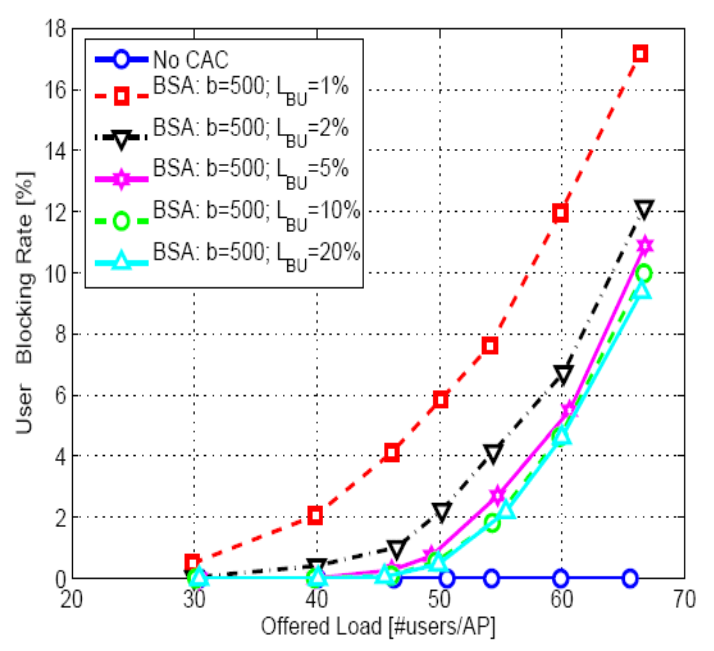

20: User blocking rate, buffer capacity of 500 packets.

As observed with the average FER, the user blocking rate increase with the offered load. In the light of the blocking threshold, the curves for the more restrictive blocking thresholds correspond to higher values of blocking rate. This happens because of the faster action of the blocking mechanism in these cases. Comparing the three figures, the 


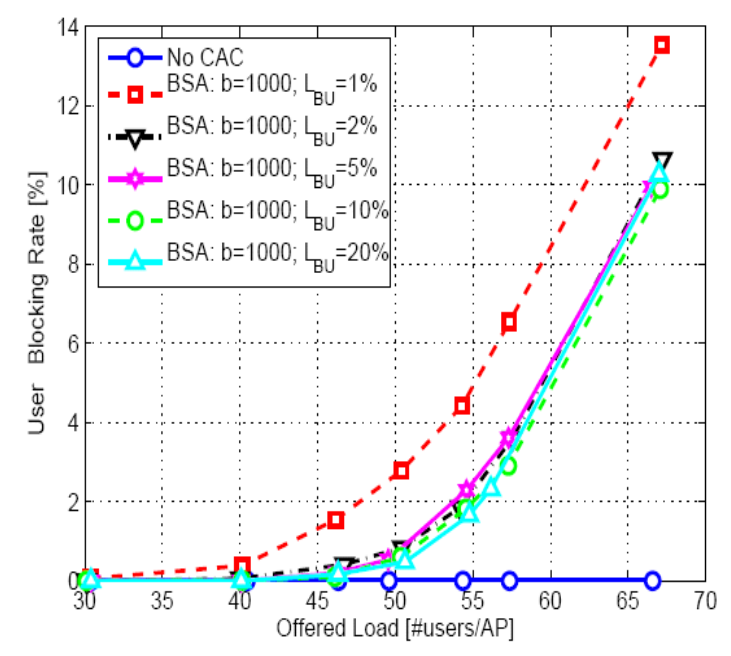

21: User blocking rate, buffer capacity of 1000 packets.

user blocking rate have a tendency to decrease when the AP's buffer capacity storage gets larger.

The satisfaction curves summarizes all the results presented before for the BSA since theses curves take into account the QoS limits for the VoIP service and the user blocking metric. The percentages of satisfied users are presented in figures 22, 23 and 24 for the three values of buffer capacity evaluated in this work, 300, 500 and 1000 packets, respectively. The $90 \%$ line is highlighted since we assume this value as threshold for defining the system capacity.

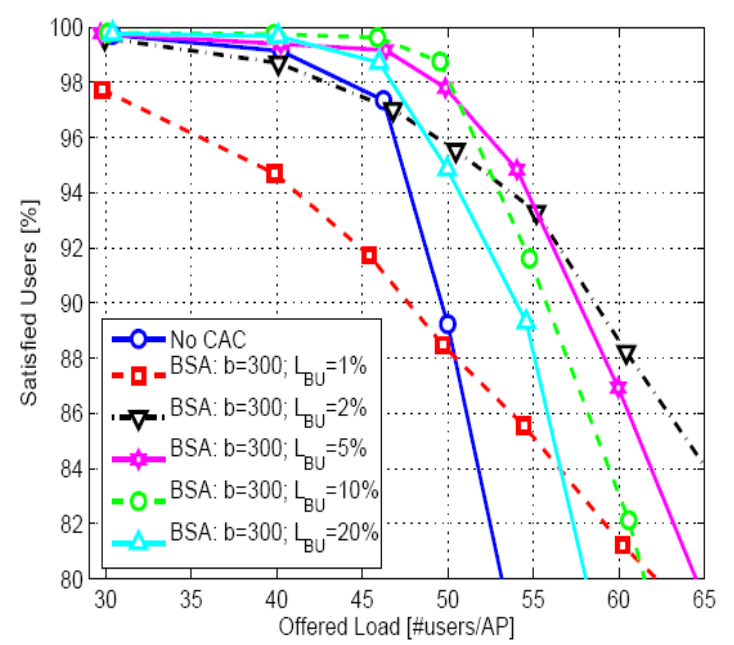

22: Percentage of satisfied users, buffer capacity of 300 packets.

The figures show the same tendency in the light of the blocking threshold. The curves for the low value of blocking thresholds (e.g. 1\%), indicate a worse performance for lower offered loads, but the strong declivity point of these curves happen at higher load. We may exploit the present of slower decrease for higher loads in order to obtain better network capacity. We have to consider that there exists a tradeoff between blocking rate threshold and buffer capacity to be

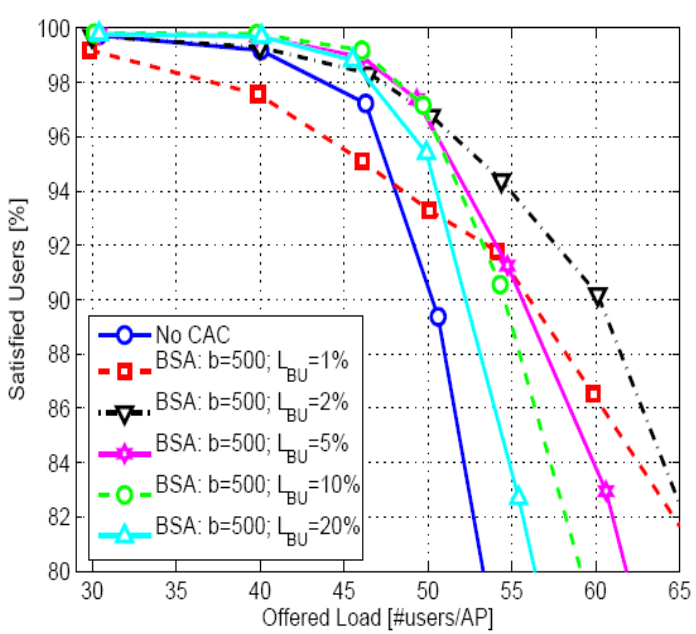

23: Percentage of satisfied users, buffer capacity of 500 packets.

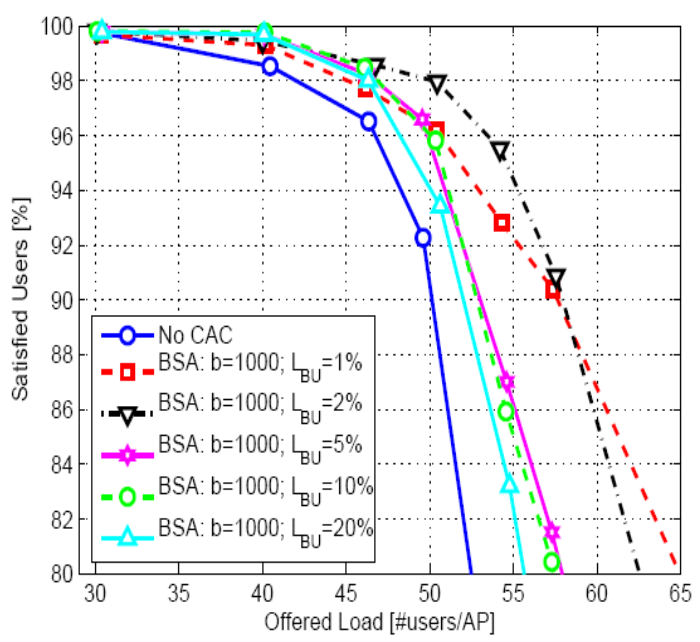

24: Percentage of satisfied users, buffer capacity of 1000 packets.

explored.

It is important to mention that the reference satisfaction curves, i. e., those ones in which no CAC mechanism was used, almost suffer no changes for the full range of storage capacity evaluated in this work.

We consider that the capacity limit is reached when a percentage of $90 \%$ of the users are satisfied. Among the three buffer sizes evaluated, the higher system capacity was reached with a buffer of 500 packets. In this case, and making use of a blocking threshold $L_{B U}$ of $2 \%$ we have obtained a capacity gain of about 20\%, supporting an offered loads up to 60 users/AP, as seen in figure 23 .

Last, but not least, it is important to notice that buffer and system stability was observed throughout our extensive simulation campaign.

\section{CONCLUSIONS}

In this work we have assessed the performance evaluation of three CAC mechanisms for the VoIP service in WLAN. 
Two of them, one based on a theoretical equation (EQA) and the other based on the channel busyness ratio (CBA), were found in the specialized literature. The third one, based on the AP's buffer utilization (BSA), corresponds to the innovative proposal of this work.

Both the EQA and the CBA achieves the basic objective of a CAC mechanism which is to guarantee the quality of service of all ongoing calls. However, this objective is achieved at the cost of system capacity. These algorithms are highly conservative in the light of the resource utilization and provide poor performance in terms of system capacity. They allocate too much resources to the VoIP flows, overprotecting the ongoing calls and causing the network's sub-utilization, without taking profit of the VoIP's inherent intermittence.

The BSA in its turn has practically eliminated the "avalanche effect" while achieving the best gains in terms of capacity and resource allocation when comparing with all the algorithms evaluated. Additionally, the practical implementation of the BSA is very simple and the decision metric is readily available at the access point.

We can further conclude that quality of service guarantees are possible for VoIP operating over WLAN by means of call admission control algorithms. Additionally, we have proposed a feasible and efficient CAC algorithm that not only leads to QoS satisfaction but also increases system capacity.

\section{ACKNOWLEDGMENTS}

We would like to thank Leonardo S. Cardoso for participating in several useful discussions that helped to improve this work. We also would like to thank the anonymous reviewers for several important suggestions that improved this paper.

\section{REFERENCES}

[1] P. Mehta and S. Udani, "Voice over IP," IEEE Potentials, vol. 20, no. 4, pp. 36 - 40, November 2001.

[2] IEEE, "IEEE 802.11 Standard: Wireless LAN Medium Access Control (MAC) and Physical Layer (PHY) Specifications," IEEE," Standard, August 1999.

[3] L. Cai, X. Shen, J. W. Mark, and Y. Xiao, "Voice Capacity Analysis of WLAN with Unbalanced Traffic," Second International Conference on Quality of Service in Heterogeneous Wired/Wireless Networks, pp. 9 9, August 2005.

[4] N. Hegde, A. Proutière, and J. Roberts, "Evaluating the voice capacity of 802.11 WLAN under distributed control," IEEE Workshop on Local and Metropolitan Area Networks, pp. 1 - 6, September 2005.

[5] D. P. Hole and F. A. Tobagi, "Capacity of an IEEE 802.11b wireless LAN supporting VoIP,' IEEE International Conference on Communications, vol. 1, pp. 196 - 201, June 2004.

[6] W. Wang, S. C. Liew, and V. O. K. Li, "Solutions to Performance Problems in VoIP over a 802.11 Wireless LAN," IEEE Transactions on Vehicular Technology, vol. 54, no. 1, pp. 366 - 384, January 2005.

[7] M. Elaoud and P. Agrawal, "Voice Capacity in IEEE 802.11 Networks," 15th IEEE International Symposium on Personal, Indoor and Mobile Radio Communications-PIMRC'2004, vol. 1, pp. 78 - 82, September 2004.

[8] S. Garg and M. Kappes, "Admission Control for VoIP Traffic in IEEE 802.11 Networks," IEEE Global Telecommunications Conference, vol. 6, pp. 3514 - 3518, December 2003.

[9] D. Chen, S. Garg, M. Kappes, and K. S. Trivedi, "Supporting VBR VoIP Traffic in IEEE 802.11 WLAN in PCF Mode," OPNETWork, August 2002.

[10] M. Veeraraghavan, N. Cocker, and T. Moors, "Support of voice services in IEEE 802.11 wireless LANs," IEEE INFOCOM, vol. 1, pp. 488 497, April 2002.
[11] R. O. Baldwin, N. J. Davis, S. F. Midkiff, and R. A. Raines, "Packetized voice transmission using RT-MAC, a wireless real-time medium access control protocol," Mobile Computing and Communications Review, pp. $11-25$, July 2001.

[12] T. Hiraguri, T. Ichikawa, M. Iizuka, and M. Morikura, "Novel Multiple Access Protocol for Voice Over IP in Wireless LAN," Seventh International Symposium on Computers and Communications, pp. 517 - 523, July 2002.

[13] A. Banchs, X. Perez, M. Radimirsch, and H. J. Stuttgen, "Service differentiation extensions for elastic and real-time traffic in 802.11 wireless LAN," IEEE Workshop on High Performance Switching and Routing, pp. 245 - 249, May 2001.

[14] D. Gu and J. Zhang, "A new measurement-based admission control method for IEEE 802.11 wireless local area networks," IEEE Proceedings on Personal, Indoor and Mobile Radio Communications, vol. 3, pp. 2009 - 2013, September 2003.

[15] Y.-L. Kuo, C.-H. Luand, E. H.-K. Wu, and G.-H. Chen, "An admission control strategy for differentiated services in IEEE 802.11," IEEE Global Telecommunications Conference, vol. 2, pp. 707 - 712, December 2003.

[16] Y. Xiao, H. Li, and S. Choi, "Protection and guarantee for voice and video traffic in IEEE 802.11e wireless LANs," IEEE INFOCOM, vol. 3, pp. $2152-2162$, September 2004.

[17] Y. Xiao and H. Li, "Voice and video transmissions with global data parameter control for the IEEE 802.11e enhance distributed channel access," IEEE Transactions on Parallel and Distributed Systems, vol. 15, no. 11 , pp. 1041 - 1053, November 2004.

[18] W. F. Fan, D. H. Tsang, and B. Bensaou, "Admission control for variable bit rate traffic using variable service interval in IEEE 802.11e WLANs," International Conference on Computer Communications and Networks, pp. $447-453,2004$

[19] D. Gao, J. Cai, and K. N. Ngan, "Admission control in IEEE 802.11e wireless LANs," IEEE Network, vol. 19, no. 4, pp. 6 - 13, August 2005.

[20] B. Makarevitch, "Scheduling and admission control for 802.11e hybrid coordinator," IEEE Vehicular Technology Conference, vol. 3, pp. 2071 - 2075, June 2005.

[21] D. Gao and J. Cai, "Admission with physical rate measurement for IEEE 802.11e controlled channel access," Communications Letters, vol. 9, no. 8 , pp. 94 - 696, August 2005.

[22] J. Freitag, N. L. S. Fonseca, and J. F. Rezende, "Mecanismos de controle de admissão baseado em medições em redes IEEE 802.11e," XXI Simpósio Brasileiro de Telecomunicações, September 2004.

[23] S. Garg and M. Kappes, "Can I Add a VoIP Call?" IEEE International Conference on Communications, vol. 2, pp. 779 - 783, May 2003.

[24] H. Zhai, J. Wang, and Y. Fang, "Providing statistical QoS guarantee for voice over IP in the IEEE 802.11 wireless LANs," IEEE Wireless Communications, vol. 13, no. 1, pp. 36 - 43, February 2006

[25] M. R. Dehkordi, K. Chandrashekar, and J. S. Baras, "Analysis of delay properties and admission control in 802.11 networks," International Conference on Wireless Networks, Communications and Mobile Computing, vol. 1, pp. 674 - 679, June 2005.

[26] D. J. Goodman, "Efficiency of packet reservation multiple access," IEEE Transactions on Vehicular Technology, vol. 40, no. 1, pp. 170-176, February 1991.

[27] IETF, "RTP: A transport protocol for real-time applications," The Internet Engineering Task Force, Tech. Rep. RFC 3550, 2003.

[28] _ - "User datagram protocol," The Internet Engineering Task Force, Tech. Rep. RFC 768, 1980.

[29] _ " "Internet protocol," The Internet Engineering Task Force, Tech. Rep. RFC 791, 1981.

[30] ITU-T, "ITU-T G.114 - One-way transmission time," Tech. Rep., September 2003

[31] T. J. Kostas, M. S. Borella, lkhlaq Sidhu, G. M. Schuster, J. Grabiec, and J. Mahler, "Real-time voice over packet-switched networks," IEEE Network, vol. 12, no. 1, pp. 18 - 27, January 1998.

[32] IEEE, "Suplement to IEEE 802.11 Standard: Wireless LAN Medium Access Control (MAC) and Physical Layer (PHY) Specifications: High-Speed Physical Layer Extension in the 5GHz Band," IEEE," Standard, September 1999.

[33] J. Geier, "The State of Wireless LANs," 2004. [Online]. Available: http://cnscenter.future.co.kr/resource/hot-topic/wlan/Intel_SR_071104.pdf

[34] A. Doufexi, S. Armour, B.-S. Lee, A. Nix, and D. Bull, "An evaluation of the performance of IEEE 802.11a and 802.11g wireless local area networks in a corporate office environment," IEEE International Conference on Communications, vol. 2, pp. 1196 - 1200, May 2003.

[35] J. C. Chen and J. M. Gilbert, "Measured Performance of $5-\mathrm{GHz} \quad 802.11 \mathrm{a} \quad$ Wireless LAN Systems," Atheros 
Communications, Inc., Tech. Rep., August 2001. [Online]. Available: www.misericordia.edu/users/srpat/dps2004/AtherosRangeCapacityPaper.pdf

[36] T. Rappaport, Wireless Communications: Principles and Practice, 2nd ed., ser. Communications Enginnering and Emerging Technologies. Prentice Hall, 2002.

[37] P. Alzén, "Multi-Cell Performance of IEEE 802.11a Wireless LANs," Master's thesis, LULEA University of Technology, January 2004. 